\title{
The dosage and administration of long-term intrathecal baclofen therapy for severe spasticity of spinal origin
}

\author{
Osamu Kawano ${ }^{1} \cdot$ Muneaki Masuda $\mathbb{( i d}^{1} \cdot$ Tsuneaki Takao $^{1} \cdot$ Hiroaki Sakai $^{1} \cdot$ Yuichiro Morishita $^{1} \cdot$ Tetsuo Hayashi $^{1}$. \\ Takayoshi Ueta ${ }^{1} \cdot$ Takeshi Maeda $^{1}$
}

Received: 11 March 2018 / Revised: 25 April 2018 / Accepted: 2 May 2018 / Published online: 12 June 2018

(c) International Spinal Cord Society 2018

\begin{abstract}
Study design Retrospective chart audit.

Objective To indicate the appropriate baclofen dosage to control severe spasticity of spinal origin and to develop the optimal administration protocol for long-term intrathecal baclofen (ITB) therapy.

Setting Department of Orthopaedic Surgery, Spinal Injuries Center, Japan.

Methods Thirty-four people with spasticity of various spinal origins who were consistently treated at our hospital were included. The median follow-up period was 6 years and 11 months. Measures of Ashworth score were taken before and after surgical implant of baclofen pump. We decided not to increase the baclofen dosage after the Ashworth score reached 1 . We recorded the control of spasticity, changes in the baclofen dose, and the incidence of complications.

Results The average Ashworth score was 3.31 (1.75-4.0) before implant surgery, 1.38 (1.0-2.25) after implant surgery, and $1.39(1.0-2.25)$ at the final follow-up, while the average baclofen dose (therapeutic/optimal dose) was $230.6 \mu \mathrm{g} / \mathrm{day}$ $(50-450)$. The incidence of each complication was as follows: $8.8 \%(n=3)$ catheter-related, $2.9 \%(n=1)$ pump-related and $5.9 \%(n=2)$ drug tolerance. No patients experienced withdrawal syndrome. Dose fluctuation with changes in the pathology of the original disease was observed in three cases.

Conclusions The usage of the Ashworth score as a guide for dose adjustment was found to be a good objective indicator for ITB therapy. The administration based on this objective indicator made it possible to effectively manage patients with a relatively low dose of baclofen and a low rate of drug-related complications.
\end{abstract}

\section{Introduction}

Intrathecal baclofen (ITB) therapy has become popular as a breakthrough treatment for severe spasticity. In Japan, a specialized lecture on ITB therapy is required and all cases are registered, ensuring the regular follow-up of patients. The incidence of complications within a short period after implantation surgery was found to be low in comparison with previous reports [1]. ITB therapy must safely control spasticity

Electronic supplementary material The online version of this article (https://doi.org/10.1038/s41393-018-0153-4) contains supplementary material, which is available to authorized users.

Osamu Kawano

orthosic@orange.ocn.ne.jp

1 Department of Orthopaedic Surgery, Spinal Injuries Center, Fukuoka, Japan over a long period of time; hence, it is extremely important to report the long-term outcomes of such cases. However, most studies on the outcomes of ITB therapy have so far included relatively short follow-up periods. The proportion of young people with spinal cord injuries who exhibit severe spasticity is also high, suggesting that strict follow-up and the accumulation of data over a long period are extremely important issues. In assessing the outcomes of ITB therapy, it is also necessary to investigate the surgical and device-related complications as well as the effects of the long-term administration of baclofen on spasticity and the associated side effects.

At our facility, since the same treatment team is responsible for patients throughout their treatment from implant surgery to long-term follow-up, continuous follow-up investigations of all cases are therefore possible [2]. The purpose of this study is to report the long-term outcomes of ITB therapy in patients who received consistent treatment from implantation to long-term follow-up at a single facility and to develop the optimal long-term administration protocol. 


\section{Methods}

The subjects were 34 consecutive patients who underwent treatment at our hospital from the time of screening tests to implant surgery at our hospital followed by dose adjustment and pump refill, with a follow-up of $\geq 2$ years. The median follow-up period was 6 years and 11 months (2-14 years). The causes of severe spasticity included spinal cord injury (SCI) $(n=29)$, hereditary spastic paraplegia (HSP) $(n=3)$, multiple sclerosis (MS) $(n=1)$ and HTLV-1 associated myelopathy $(\mathrm{HAM})(n=1)$.

In determining the dose of baclofen to control spasticity, the degree of spasticity (Ashworth score [3, 4]) was used as the only guide, and attention was paid not to increase the dosage for the purpose of controlling pain. The spasticity was rated not by the authors but by the physical therapist, who had received a thorough education with regard to spinal cord injuries. Our main strategy for managing spasticity was to allow a small amount of spasticity to remain, so that we could prevent the lower limbs from becoming completely flaccid [5].

In all cases, changes in the Ashworth score before and after treatment (before surgery, within 1 month after implant surgery and at the final follow-up) and changes in the baclofen dose were examined to evaluate the control of spasticity. Statistical analyses concerning changes in the Ashworth score were performed in order to confirm that the spasticity was significantly reduced and the effectiveness of the ITB therapy was maintained. An analysis of variance (ANOVA) was used to analyze the results statistically. A $P$ value of $<0.05$ was considered to indicate statistical significance. In addition, all complications were investigated.

\section{Results}

We examined the baclofen dose of each patient in whom spasticity could be controlled over a long period of time and found that the long-term dose was stable in most of cases, while it fluctuated significantly in a small number of cases. Catheter-related trouble occurred in three cases, including catheter microleaks $(n=2)$ and migration of the catheter to the subdural space $(n=1)$. Dose fluctuation was defined as a dose increase of $>100 \mu \mathrm{g}$ in the daily baclofen dose per year in the absence of device-related trouble. Patients whose dose displayed fluctuation were classified into the fluctuation group $(n=5)$, and all others were classified into the stable group $(n=26)$. The indicator, an $>100 \mu \mathrm{g}$ increase in the daily baclofen dose over the course of one year, was set according to the definition of drug tolerance described by Heelta et al [6]. In three of the five cases in the fluctuation group, the fluctuation was associated with change in the original disease such as disease progression related to multiple sclerosis $(n=1)$, severe myasthenia gravis concomitant with cervical cord injury $(n=1)$, and spasticity relief related to an improvement of cervical cord injury $(n=1)$ in which the dosage was decreased as the spasticity lessened and the muscle strength declined. In the remaining two cases in the fluctuation group, the increased dosage was considered to be due to the development of drug tolerance.

There were two cases involving complications that required revision surgery; surgical site infection (SSI) $(n=1)$ and pump exchange due to pump malfunction $(n=1)$. As regard to the case of pump malfunction and two cases of catheter microleaks, surgery was performed to replace the pump or catheter immediately after the issues were identified. There were no cases of withdrawal syndrome. Migration of the catheter to the subdural space was diagnosed in the 6 week after the initial surgery, and the catheter was immediately replaced by revision surgery and the position of the catheter was confirmed by an intraoperative fluoroscopic contrast study. In one case of SSI, we removed the pump and catheter at once. We performed reimplantation surgery after 4 months when the infection had completely resolved. At the time of removal, the baclofen dosage was gradually decreased and finally interrupted as to take care not to cause withdrawal syndrome.

Thus, the complications over the long-term course were as follow: catheter-related $8.8 \%(n=3)$; pump-related $2.9 \%$ $(n=1)$; SSI $2.9 \%(n=1)$, and drug tolerance $5.9 \%(n=2)$ (Table 1). There were no cases of withdrawal syndrome or overdose. Both catheter-related and pump-related complications occurred due to the usage of old types of catheters and pumps; thus far, no issues have been experienced in association with the use of current-generation devices.

The average Ashworth score over the long-term course was 3.31 (1.75-4.0) before surgery, 1.38 (1.0-2.25) after surgery, and $1.39(1.0-2.25)$ at the end of all follow-up periods (Fig. 1). Significant differences were observed in the average Ashworth score before and after surgery and in the average Ashworth score before surgery and at the end of all follow-up periods. The average baclofen dose during long-term administration was $230.6 \mu \mathrm{g} /$ day (50-450 $\mu \mathrm{g} /$ day) (Fig. 2).

\section{Discussion}

Severe spasticity, which is often associated with spinal cord lesions, requires treatment, as it reduces the patient's activities of daily living (ADL) and significantly impairs the quality of life (QOL). Although the effect of oral baclofen is not sufficient, ITB therapy, which is now widely performed, is a drastic and extremely effective treatment that allows baclofen to be continuously administered directly into the subarachnoid space by an implantable pump [7].

In order to safely and effectively continue ITB therapy, appropriate surgical implantation procedures and strict 
Table 1 Changes of Ashworth score, dose of baclofen and complications in long-term ITB therapy

\begin{tabular}{|c|c|c|c|c|c|c|c|c|}
\hline Author & $\begin{array}{l}\text { Follow-up } \\
\text { (years) }\end{array}$ & $\begin{array}{l}\text { No. of } \\
\text { patients }\end{array}$ & $\begin{array}{l}\text { AS } \\
\text { (pre) }\end{array}$ & $\begin{array}{l}\text { AS } \\
\text { (post) }\end{array}$ & $\begin{array}{l}\text { Dose }(\mu \mathrm{g} / \\
\text { day) }\end{array}$ & $\begin{array}{l}\text { Catheter problems } \\
(\%)\end{array}$ & $\begin{array}{l}\text { Pump problems } \\
(\%)\end{array}$ & $\begin{array}{l}\text { Tolerance } \\
(\%)\end{array}$ \\
\hline Ordia et al. [8] & 6.1 & 131 & 4.2 & 1.3 & $134 \sim 277$ & 15 & 2 & 1.5 \\
\hline Plassat et al. [9] & 4 & 40 & 1.8 & 1.8 & & 58 & 42 & NA \\
\hline Zahavi et al. [10] & 7.1 & 21 & 2.8 & 0.9 & 290 & 23 & 3 & NA \\
\hline Heetla et al. [11] & 3.2 & 37 & 3.7 & 2.4 & 350 & 27 & 3 & 22 \\
\hline Draulans et al. [12] & 6.2 & 130 & NA & NA & 504 & 60 & 6 & NA \\
\hline Mathur et al. [13] & 14.7 & 24 & NA & NA & 627 & NA & NA & NA \\
\hline Boster et al. [5] & NA & NA & NA & NA & 400 (mean) & NA & NA & NA \\
\hline $\begin{array}{l}\text { Kawano et al. } \\
\text { (present study) }\end{array}$ & 6.9 & 34 & 3.31 & 1.39 & 230.6 & 8.8 & 2.9 & 5.7 \\
\hline
\end{tabular}

$A S$ Ashworth score, NA not available

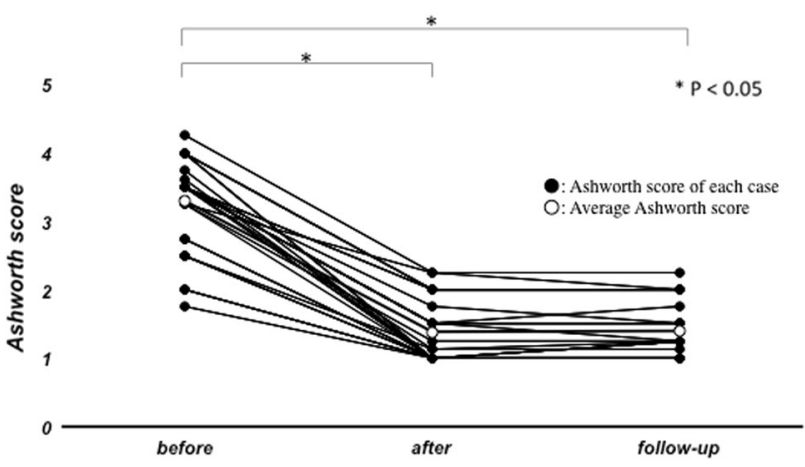

Fig. 1 Ashworth score over the long-term course $(n=34)$ : The average Ashworth score over the long-term course was 3.31 (1.75-4.0) before surgery, $1.38(1.0-2.25)$ after surgery, and $1.39(1.0-2.25)$ at the end of all follow-up periods

patient management are important [2]. Various issues related to drugs and implanted devices can be expected to occur over the course of long-term follow-up. An understanding of the clinical condition of the patient before treatment as well as regular observation of how the spasticity changes as a result of treatment can lead to the early detection of trouble and complications, thereby enabling the continuation of safe treatment [5]. We have set certain criteria for drug dose adjustment to allow for consistent treatment to be delivered. Specifically, when adjusting the dose, we decided to allow a certain degree of spasticity to remain in the lower limbs and do not to increase the dose further in order to reduce the spasticity-related pain once the lower limbs became flaccid. In addition, to facilitate the consistent performance of surgery, one of three senior surgeons (OK, MM, and TU) was in charge of all implant surgeries. The results of treatment under this policy were reflected in the incidence of complications and the transition of the dose in the present study.

Three incidents of catheter-related trouble occurred $(8.8 \%)$ over the course of the long-term follow-up; this frequency was relatively low in comparison to previous reports [8-13] (Table 1). Furthermore, an Ashworth score of approximately

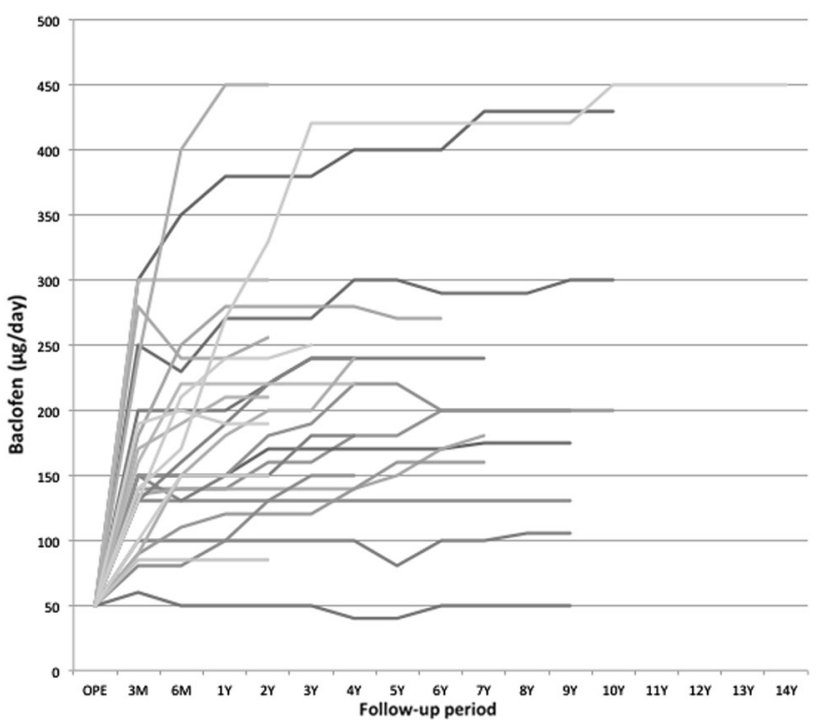

Fig. 2 ITB dose in the stable group $(n=26)$ : The average baclofen dose during long-term administration was $230.6 \mu \mathrm{g} /$ day (50-450 $\mu \mathrm{g} /$ day)

1.39 was maintained with an average dose of $230.6 \mu \mathrm{g} /$ day, thus indicating that spasticity was well-controlled at a relatively low dose in comparison to the results described in previous reports [5, 8-13] (Table 1). Attempting to completely eliminate spasticity in order to control various types of pain by increasing the baclofen dose can easily lead to an excessive increase in the dosage. This is associated with the risk of withdrawal syndrome, which occurs due to device-related trouble, and may increase the risk of drug tolerance [2]. Therefore, developing specific criteria and avoiding unnecessary dosage increases may lead to good long-term outcomes.

Saulino et al. [2] stated that communication among all members of the treatment team is essential for achieving a successful patient outcome. They also stated that the combination of a well-organized ITB team and a committed patient/family will result in the vast majority of patients being satisfied with this therapy. However, the present study only included 34 patients who received continuous 
treatment at the same hospital from the introduction of ITB therapy. It may be necessary to increase the number of patients undergoing long-term follow-up without any withdrawal and to report the outcomes over a longer period; a well-organized multi-center ITB team would be wellsuited to carrying out such a study [2].

There were two cases in which drug tolerance was suspected in this survey. In both cases, the suspected drug tolerance occurred after more than 6 years. The patients' progress was good in a simple continuous drug infusion mode; however, a sudden loss of efficacy was observed. As it was impossible to control the patients' spasticity completely, even by increasing the baclofen dosage, pulsatile bolus infusion was used to achieve spasticity control [6]. Specifically, in the first case, in addition to the basal dose of $5.5 \mu \mathrm{g} / \mathrm{day}, 30 \mu \mathrm{g}$ of regularly scheduled boluses were administered at 6-h intervals. In the second case, boluses of the same dose were administered at 8-h intervals. Spasticity control was achieved in both cases. Attempting a drug holiday of several weeks does not seem realistic, as the patient would suffer from severe spasticity over the course of the drug holiday. Periodic bolus delivery with a very small basal dose might create a situation similar to a short drug holiday in the $\mathrm{GABA}_{\mathrm{B}}$ receptors in the spinal cord, resulting in resensitization of the receptors. However, there is no consensus or unified definition regarding drug tolerance, and therefore no approach to solving this problem has yet been established. It is thought that these problems, which may be very important going forward, will be solved by having a consistent medical team conducting regular observations over a long follow-up period [2]. This study is limited by its use of retrospective chart audits, small number of patients, and single-center setting. Further research in a multicenter prospective study or highvolume review may help to develop a safe and optimal protocol for long-term ITB therapy.

\section{Conclusions}

Severe spasticity of spinal origin was well controlled with relatively low doses of baclofen, the dosage of which was determined based on objective indicators, with a low rate of complications. The Ashworth score was used as a guide for dose adjustment. The baclofen dosage was not increased after the average Ashworth score reached 1.

Funding The manuscript submitted does not contain information about medical device(s)/drug(s). No funds were received in support of this work. No benefits in any form have been or will be received from a commercial party related directly or indirectly to the subject of this manuscript.

\section{Compliance with ethical standards}

Statement of ethics This study was approved by the Ethics Review Board of the Spinal Injuries Center. This article does not contain any studies with human participants or animals performed by any of the authors. Informed consent was obtained from all of the patients in the present study.

Conflict of interest The authors declare that they have no conflict of interest.

\section{References}

1. Taira T, Ueta T, Katayama Y, Kimizuka M, Nemoto A, Mizusawa $\mathrm{H}$, et al. Rate of complications among the recipients of intrathecal baclofen pump in Japan: a multicenter study. Neuromodulation. 2013;16:266-72.

2. Saulino M, Anderson DJ, Doble J, Farid R, Gul F, Konrad P, et al. Best practices for intrathecal baclofen therapy: troubleshooting. Neuromodulation. 2016;19:632-41.

3. Ashworth B. Preliminary trial of carisoprodol in multiple sclerosis. Practitioner. 1964;192:540-2.

4. Boster AL, Bennett SE, Bilsky GS, Gudesblatt M, Koelbel SF, McManus M, et al. Best practices for intrathecal baclofen therapy: screening test. Neuromodulation. 2016;19:616-22.

5. Boster AL, Adair RL, Gooch JL, Nelson ME, Toomer A, Urquidez $\mathrm{J}$, et al. Best practices for intrathecal baclofen therapy: dosing and long-term management. Neuromodulation. 2016;19:623-31.

6. Heetla HW, Staal MJ, van Laar T. Tolerance to continuous intrathecal baclofen infusion can be reversed by pulsatile bolus infusion. Spinal Cord. 2010;48:483-6.

7. Saulino M, Ivanhoe CB, McGuire JR, Ridley B, Shilt JS, Boster AL. Best practices for intrathecal baclofen therapy: patient selection. Neuromodulation. 2016;19:607-15.

8. Ordia JI, Fischer E, Adamski E, Chagnon KG, Spatz EL. Continuous intrathecal baclofen infusion by a programmable pump in 131 consecutive patients with severe spasticity of spinal origin. Neuromodulation. 2002;5:16-24.

9. Plassat R, Perrouin Verbe B, Menei P, Menegalli D, Mathe JF, Richard I. Treatment of spasticity with intrathecal baclofen administration: long-term follow-up, review of 40 patients. Spinal Cord. 2004;42:686-93.

10. Zahavi A, Geertzen JH, Middel B, Staal M, Rietman JS. Long term effect (more than five years) of intrathecal baclofen on impairment, disability, and quality of life in patients with severe spasticity of spinal origin. J Neurol Neurosurg Psychiatry. 2004;75:1553-7.

11. Heetla HW, Staal MJ, Kliphuis C, van Laar T. The incidence and management of tolerance in intrathecal baclofen therapy. Spinal Cord. 2009;47:751-6.

12. Draulans N, Vermeersch K, Degraeuwe B, Meurrens T, Peers K, Nuttin B, et al. Intrathecal baclofen in multiple sclerosis and spinal cord injury: complications and long-term dosage evolution. Clin Rehabil. 2013;27:1137-43.

13. Mathur SN, Chu SK, McCormick Z, Chang Chien GC, Marciniak $\mathrm{CM}$. Long-term intrathecal baclofen: outcomes after more than 10 years of treatment. PM \& R. 2014;6:506-13. 